\title{
MOLE: A New High-Energy Gamma-Ray Diagnostic
}

\author{
Michael J. Moran and Britton Chang
}

January 21, 1992

This is an informal report intended primarily for internal or limited external distribution. The opinions and conclusions stated are those of the author and may or may not be those of the Laboratory.

Work performed under the auspices of the U.S. Department of Energy by the

Lawrence Livermore National Laboratory under Contract W-7405-Eng-48. 


\begin{abstract}
DISCLAIMER ;
This document was prepared as an account of work sponsoref by an agency of the United States Government. Neither the United States Government nor the University of Californiz-nor any of their employees, makes any warranty, express or implied, or assumes any legal liability or responsibility for the accuracy, completeness, or usefulness of any information, apparatus, product, or process disclosed, or represents that its use would not infringe privately own rights. Reference herein to any specific commercial products, process, or service by trade name, trademark, manufacturer, or otherwise, does not necessarily constitute or imply its endorsement, recommendation, or favoring by the United States Government or the University of California. The views and opinions of authors expressed herein do not necessarily state or reflect those of the United States Government or the University of California, and shall not be used for advertising or product endorsement purposes.
\end{abstract}

This report has been reproduced directly from the best available copy.

Available to DOE and DOE contractors from the Office of Scientific and Technical Information P.O. Box 62, Oak Ridge, TN 37831

Prices available from (615) 576-8401, FTS 626-8401

Available to the public from the National Technical Information Service

U.S. Department of Commerce 5285 Port Royal Rd. Springfield, VA 22161 


\section{DISCLAIMER}

This report was prepared as an account of work sponsored by an agency of the United States Government. Neither the United States Government nor any agency Thereof, nor any of their employees, makes any warranty, express or implied, or assumes any legal liability or responsibility for the accuracy, completeness, or usefulness of any information, apparatus, product, or process disclosed, or represents that its use would not infringe privately owned rights. Reference herein to any specific commercial product, process, or service by trade name, trademark, manufacturer, or otherwise does not necessarily constitute or imply its endorsement, recommendation, or favoring by the United States Government or any agency thereof. The views and opinions of authors expressed herein do not necessarily state or reflect those of the United States Government or any agency thereof. 


\section{DISCLAIMER}

Portions of this document may be illegible in electronic image products. Images are produced from the best available original document. 


\section{MOLE: A New High-Energy $\gamma$-Ray Diagnostic*}

UCRL-ID--109712

Michael J. Moran and Britton Chang

DE92 009742

Lawrence Livermore National Laboratory

Livermore, CA 94550

January 21, 1992

\section{Introduction}

Continued interest in high-energy $\gamma$ rays associated with fusion reactions has motivated an ongoing search for simple, effective measurement techniques. Past experiments have measured $16.7-\mathrm{MeV} \gamma$ rays with Compton-magnetic spectrometers. Some measurements have been performed with threshold Cherenkov detectors with enhanced sensitivity to high-energy $\gamma$ rays. The Compton spectrometers work quite well, but they require extensive calibrations and tend to be expensive and cumbersome. The threshold Cherenkov detectors are simpler to calibrate and physically compact, but have poor spectral definition and are vulnerable to background signals. This report is to describes a new type of $\gamma$-ray detector, the MOLE, that may retain the simplicity of a threshold Cherenkov detector while still having sufficient energy discrimination to be effective for measuring high-energy $\gamma$-rays in the presence of lower-energy $\gamma$-ray fluxes.

\section{Description}

The basic intention of MOLE is to design a system that converts highenergy $\gamma$ rays $\left(E_{\gamma} \geq 15 \mathrm{MeV}\right.$ ) to a well-defined electron beam very efficiently (q.e. $\approx$ $\left.10^{-3}-10^{-4}\right)$, while converting lower energy $\gamma$ rays $\left(\mathrm{E}_{\gamma} \leq 4 \mathrm{MeV}\right)$ with extremely poor efficiency (q.e. $\ll 10^{-7}$ ). MOLE is a detector whose electron response increases rapidly with increasing $\gamma$-ray energy and has a low-energy threshold. An energydependent electron detector can enhance the energy discrimination of the system.

MOLE takes advantage of the relationships between incident particle energy and the angular widths of transmitted electron distributions for pair production, Compton scattering and Coulomb scattering (Molière scattering) of electrons in solids. Energy loss by electrons as they pass through the scattering media leads to an energy threshold.

\footnotetext{
- Work performed under the auspices of the U.S. Department of Energy by the Lawrence Livermore National Laboratory under Contract W-7405-Eng-48.
} 
The important feature of the scattering mechanisms mentioned above is that their emission distributions have angular widths that are inversely proportional to incident particle energy. Thus, high-energy particles may produce narrow, forward-directed distributions, while lower-energy particles disperse electrons through wide angular ranges. Since the peak forward intensity will be inversely proportional to the square of the distribution width, forward scattered electron beam intensities will tend to enhance higher-energy electrons in proportion to the square of their energies. If the forward-scattered fluxes are selected, and this process is repeated several times in a system, the overall relative enhancement of high-energy electron fluxes will be by the product of the enhancements for each process.

To see how this system might work, consider two beams of incident $\gamma$ rays having an energy ratio of five (e.g., $15-\mathrm{MeV}$ and 3-Mev $\gamma$ s). According to the conjecture above, the high-energy flux ratio could increase by a factor of twenty. five each time the particles are scattered and selected. A threestage system might increase the ratio by a factor of $(25)^{3}$, or over $1.5 \cdot 10^{4}$. Finally, an electron detector having sensitivity proportional to electron energy could increase the overall enhancement by another factor of five to $7.5 \cdot 10^{4}$.

\section{An Example}

These ideas were studied more closely by using the model system shown schematically Fig. 1. A collimated beam of $\gamma$ rays incident on a $0.025-\mathrm{cm}$ thick $\mathrm{U}^{238}(\gamma, \mathrm{e})$ converter produces a forwarddirected beam of electrons. Next, a "ring"collimator selects narrowangle (higher-energy) components

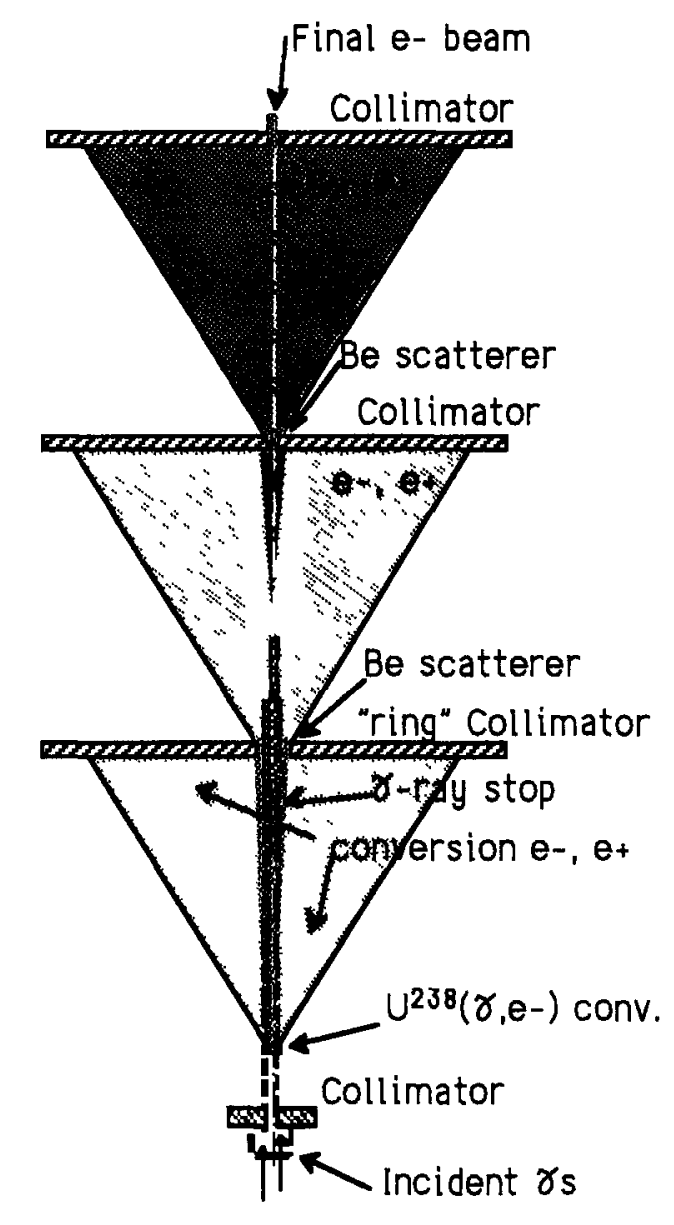

Figure 1. MOLE schematic geometry. 
of the beam and directs them to a $0.5-\mathrm{cm}$ thick Be scatterer that generates a new broadened angular distribution. Another small-angle collimator and Be scatterer enhance the high-energy character of the electron spectrum further. A final collimator selects small-angle portions of the beam before measurement by some kind of electron detector.

The behavior of this type of system has been modeled by a relatively simple computer code. 1 The code is based on a combination of analytical description of the processes mentioned above: pair production, Compton scattering, etc. The code has a "menu-driven" organization: different kinds of calculations are used to model different portions of the system. The paragraphs below discuss calculations for the system described in Fig. 1.

\section{Calculations:}

Figure 2 shows the overall results of the calculations. Here, we show electrons transmitted through the system as a function of incident $\gamma$-ray energy. The solid line shows roughly a three-decade increase in efficiency as the $\gamma$-ray energy increases from $4 \mathrm{MeV}$ to 17

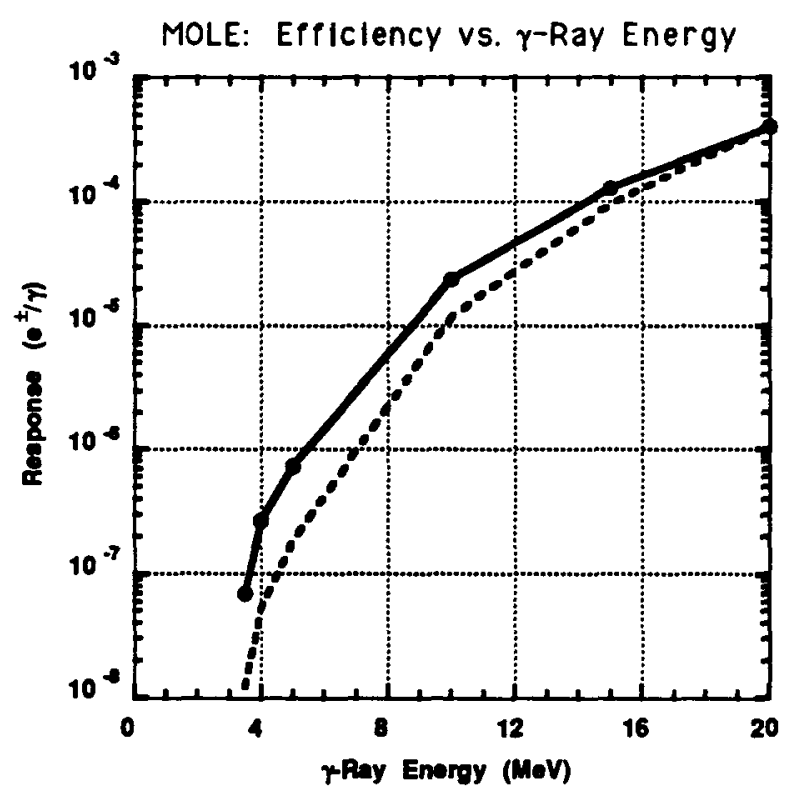

Figure 2. MOLE response vs. łray energy. MeV. An energy threshold somewhere between 3 and $4 \mathrm{MeV}$ eliminates response to lower-energy $\gamma$ rays. The dotted line suggests how the energy discrimination might be enhanced by an electron detector whose sensitivity is proportional to electron energy. If the electron detector also features gains of 10 to $10^{2}$ then the overall "quantum efficiency" of the system would be of the order of .002 to .02 for the higher $\gamma$-ray energies considered here. 


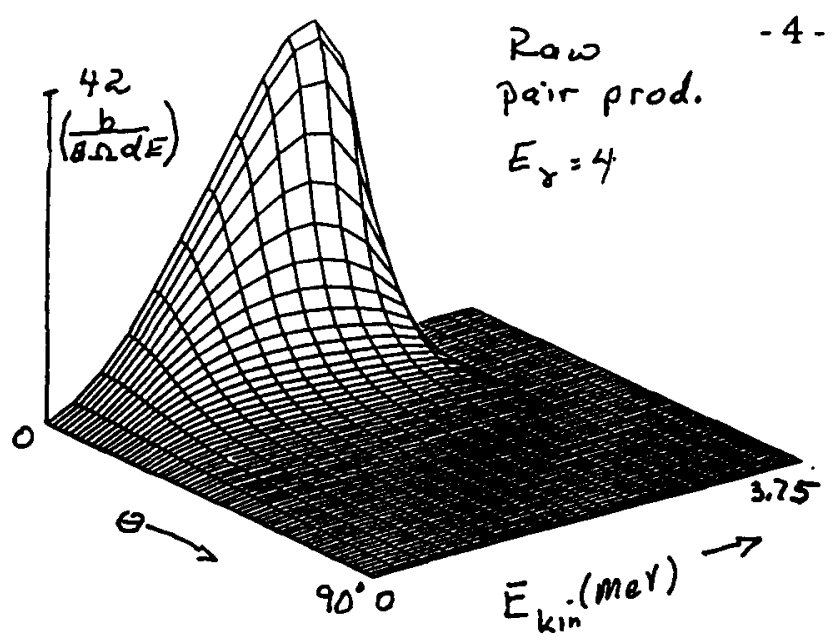

(a)

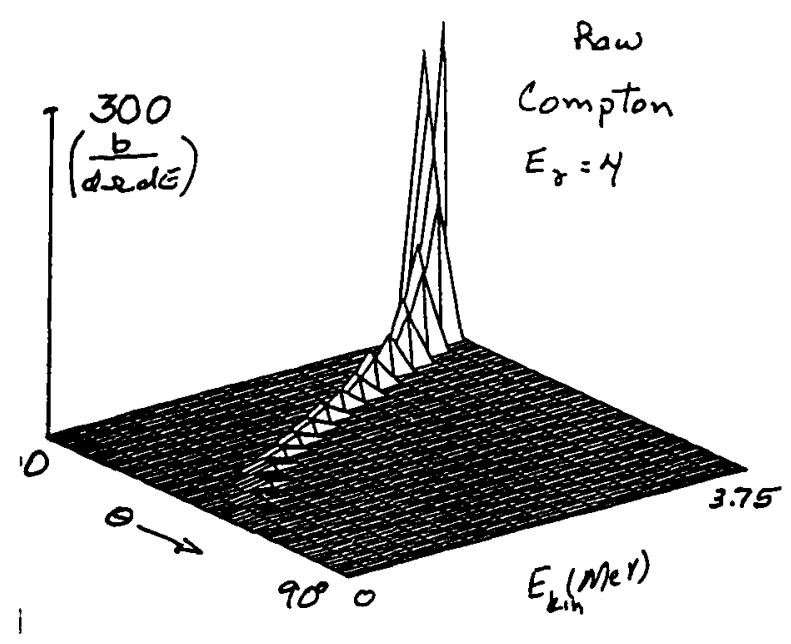

(b)
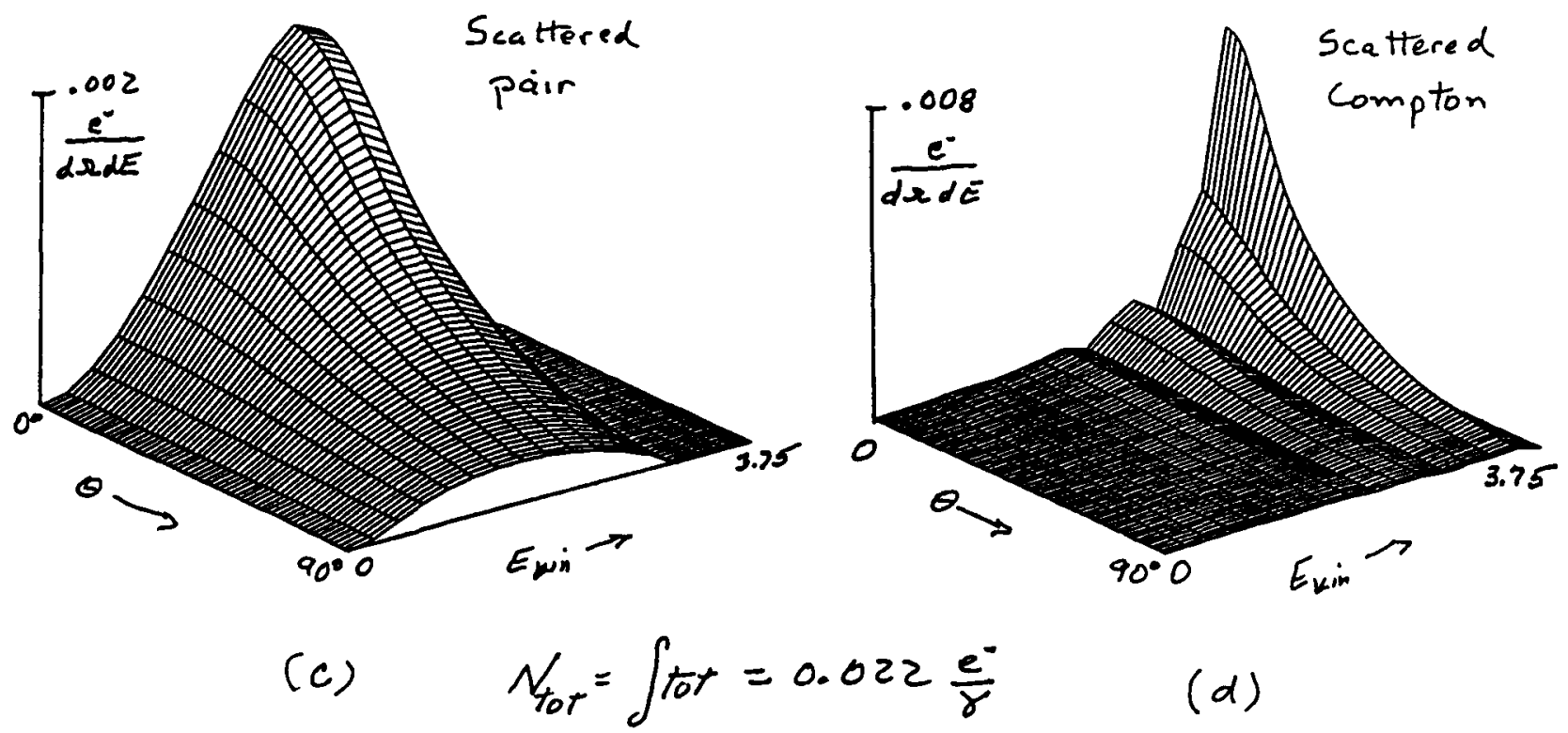

Figure 3. MOLE calculations for $E \gamma=4 \mathrm{MeV}$.

Calculations: $E_{\gamma}=4 \mathrm{MeV}$ : the ${ }^{238} \mathrm{U}$ Converter

Figure 3 shows results from calculations of electron distributions produced by the ${ }^{238} \mathrm{U}$ converter in response to $4 \mathrm{MeV} \gamma$ rays. Figures $3(\mathrm{a})$ and $3(\mathrm{~b})$ show "raw" pair production and Compton scattering cross section $\left(\sigma_{\mathrm{pp}}\right.$ and $\left.\sigma_{\mathrm{C}}\right)$ spectralangular distributions. Angle increments are $1.5^{\circ}$ and energy increments are about $0.25 \mathrm{MeV}$. For this $\psi$-ray energy the forward $\sigma_{\mathrm{C}}$ is substantially larger than $\sigma_{\mathrm{pp}}$. For the angle-energy bins used here, not every row or column of Fig. 3(b) contains non-zero elements, because of the energy-angle relationship of $\sigma_{\mathrm{C}}$. However, integration of the entire $\sigma_{\mathrm{C}}$ distribution gives the correct total cross section. 

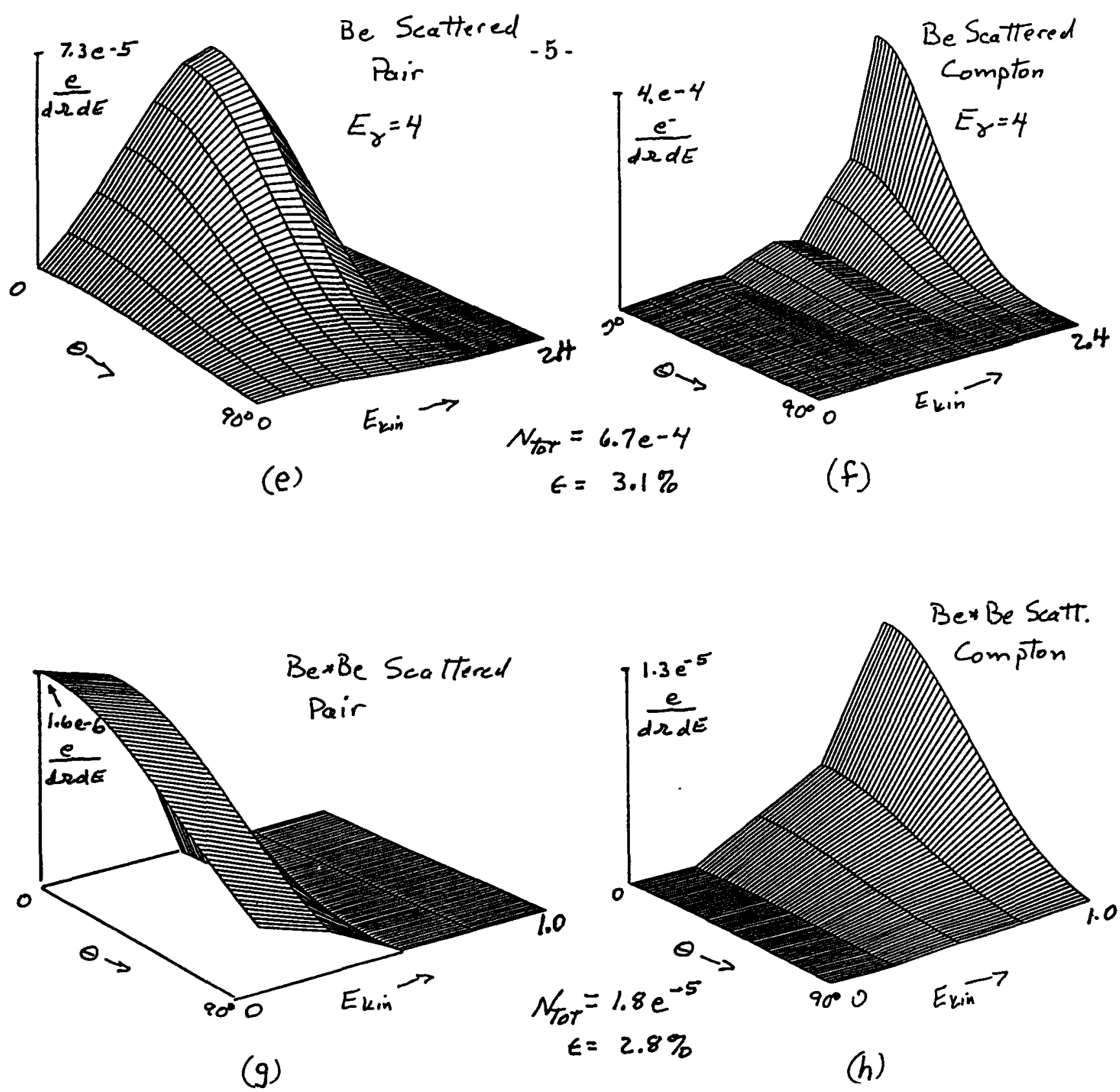

Figure 3. MOLE calculations for $E_{\gamma}=4 \mathrm{MeV}$ (cont'd).

The $(\gamma, \mathrm{e})$ conversion uses both Compton scattering and pair production. The angular widths of both processes decrease with increasing $\gamma$-ray energy. Coulomb scattering of the electrons will broaden the distributions further (in inverse proportion to electron energy) before the electrons escape the converter. The energy dependence of $\sigma_{\mathrm{pp}}$ helps to enhance the high-energy $\gamma$-ray response of the system. This approach contrasts with the design of a Compton spectrometer, which requires a well-defined angle-energy relationship for scattered electrons. With the MOLE, the energy-angle relationship is sacrificed in favor of increased $(\gamma, e)$ conversion. The $\mathrm{Z}^{2}$ dependence of pair production also is the main reason for using a high- $Z$ converter. 
The computer code folds $\sigma_{\mathrm{pp}}$ and $\sigma_{\mathrm{C}}$ with Molière scattering distributions and integrates over the thickness of the converter. This is accomplished by dividing the converter into a number $(\approx 10)$ of thin layers. The computer code folds distributions of conversion electrons from each sublayer with Molière broadening appropriate for each sublayer. Figures 3(c) and 3(d) show new pair and Compton $\mathrm{e}^{-}$distributions that reflect the effect of Molière scattering in the converter. These results show substantial broadening of the angular distributions. Electron energy loss from passage through the converter is ignored. $\mathrm{N}_{\text {tot }}$ is used as an indicator of the total scattered particle flux $\left(\mathrm{N}_{\mathrm{tot}} \approx \int\left(\sigma_{\mathrm{C}}+2 * \sigma_{\mathrm{pp}}\right)\right)$. For Fig. $3(c)$ and $3(\mathrm{~d}) \mathrm{N}_{\text {tot }}$ is $.022 \mathrm{e}^{ \pm}$per incident $\gamma$ ray.

After passing through the converter, $\gamma$ rays stop in a $\mathrm{Pb}$ "get lost". Gamma rays will pass further into the system only if they are scattered by the converter. A circular "ring" collimator selects electrons scattered into near-forward angles (e.g., $2^{\circ}-5^{\circ}$ ). Because higher-energy $\gamma$ rays produce narrower forward-directed beams of higher-energy electrons, the hope is that higher-energy electrons will be transmitted preferentially by this collimator, compared to lower-energy electrons from lower-energy $\gamma$ rays. This completes the first stage.

\section{First Be Scatterer}

After being selected by the "ring" collimator, the "re-collimated" electrons pass through the first $0.5-\mathrm{cm}$ thick Be scatterer. Here, the intent is to improve the low-energy/high-energy discrimination by forcing particles to scatter in a low- $Z$ medium that minimizes the production of extraneous ionizing radiation, such as Bremsstrahlung.

The Be scatters the electrons into new angular distributions having widths inversely proportional to energy. Figure $3(e)$ and 3( $f$ ) show the "re-broadened" distributions for "pair" and Compton electrons, respectively. Note also that the peak kinetic energy has been reduced by about $1.4 \mathrm{MeV}$. The new value for $\mathrm{N}_{\text {tot }}$ of $6.7 \cdot 10^{-4}$ indicates that $3.1 \%$ of the flux was transmitted by the "ring" collimator.

Options in the calculations were chosen to be conservative with respect to errors. The new Molière scattered distributions used the incident electron energy, ignoring the impact of energy loss on the distributions. Also, the calculations evaluate the distributions for $3^{\circ}$ to $6^{\circ}$ (because of the angle binning), rather than 
the desired $2^{\circ}$ to $5^{\circ}$. Both of these choices are conservative: they reduce the actual transmitted flux and reduce the effectiveness of energy discrimination.

In addition to Coulomb scattering, the electrons suffer energy loss as they pass through the scatterer. For $0.5-\mathrm{cm}$ Be the energy loss is about $1.4 \mathrm{MeV}$ for a wide range of electron energies. This is significant, as it establishes an energy threshold that discriminates against low-energy $\gamma$ rays.

The electrons pass through a second collimator-this time a circular onaxis collimator that selects scattered angles of about $4^{\circ}$ to $8^{\circ}$. As previously, the design is chosen to allow efficient transmission of highenergy electrons, while discriminating against lower energies. This completes the second stage.

\section{Second Be Scatterer}

The process is repeated with a second $0.5-\mathrm{cm}$ Be scatterer, where the electron distributions are "re-broadened" again. The calculations again use angle bins from $3^{\circ}$ to $6^{\circ}$, resulting in a slight overestimate of collimator efficiency and energy discrimination. The resulting distributions are shown in Figures $3(\mathrm{~g})$ and $3(\mathrm{~h})$, respectively. Note that the kinetic energy spectrum now is reduced to a peak value of about $1 \mathrm{MeV}$. The new value for $\mathrm{N}_{\text {tot }}\left(\approx 1.8 \bullet 10^{-5}\right)$ corresponds to transmission of $2.8 \%$ of the flux incident on the second collimator. Here, the pair $\mathrm{e}^{ \pm}$spectrum maximum energy is only about $0.5 \mathrm{MeV}$. The electron flux clearly is dominated by contributions from Compton scattering.

The final stage is a re-collimation that selects particles having velocities from $2^{\circ}$ to $5^{\circ}$ off-axis. The transmitted spectra are calculated by selecting angles from $3^{\circ}$ to $6^{\circ}$ from the distributions in Figs. $3(\mathrm{~g})$ and $3(\mathrm{~h})$. Again, the angle bins are not exactly correct, but the errors now are conservative, in that transmitted flux and energy discrimination will be underestimated.

\section{Calculations: $\mathrm{E}_{\boldsymbol{\gamma}}=15 \mathrm{MeV}$}

The calculations described above were repeated with $15-\mathrm{MeV} \gamma$ rays. Figures $4(\mathrm{a})$ and $4(\mathrm{~b})$ show the "raw" $\sigma_{\mathrm{pp}}$ and $\sigma_{\mathrm{C}}$ calculated for the same $0.025-\mathrm{cm} \mathrm{U}$ U38 converter. Differences associated with the higher $\psi$-ray energy are apparent: the differential cross sections have larger peak values and have much narrower forward-directed distributions. $\sigma_{\mathrm{pp}}$ now has peak values that are comparable to those for $\sigma_{\mathrm{C}}$. The $\mathrm{e}^{ \pm}$spectra extend now to almost $15 \mathrm{MeV}$ kinetic energy. 


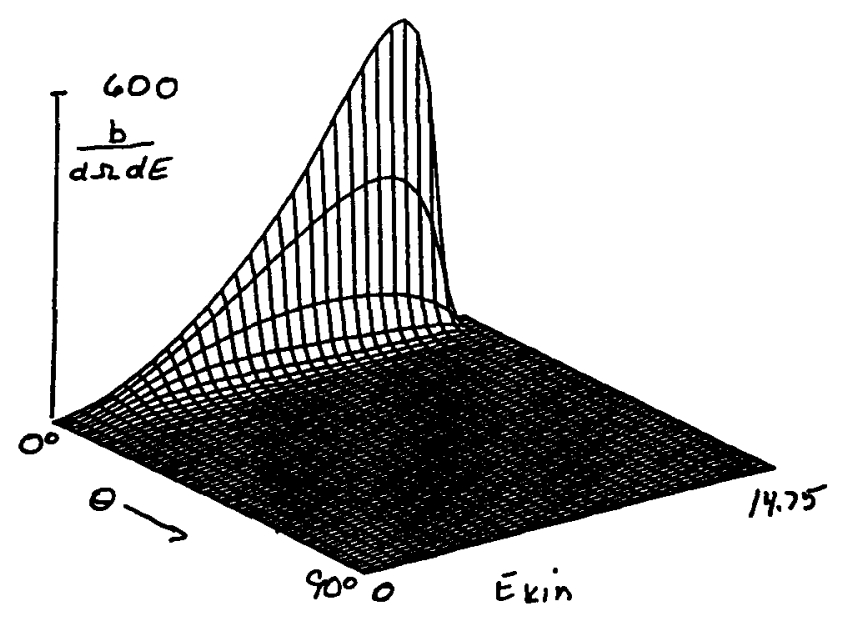

(a)

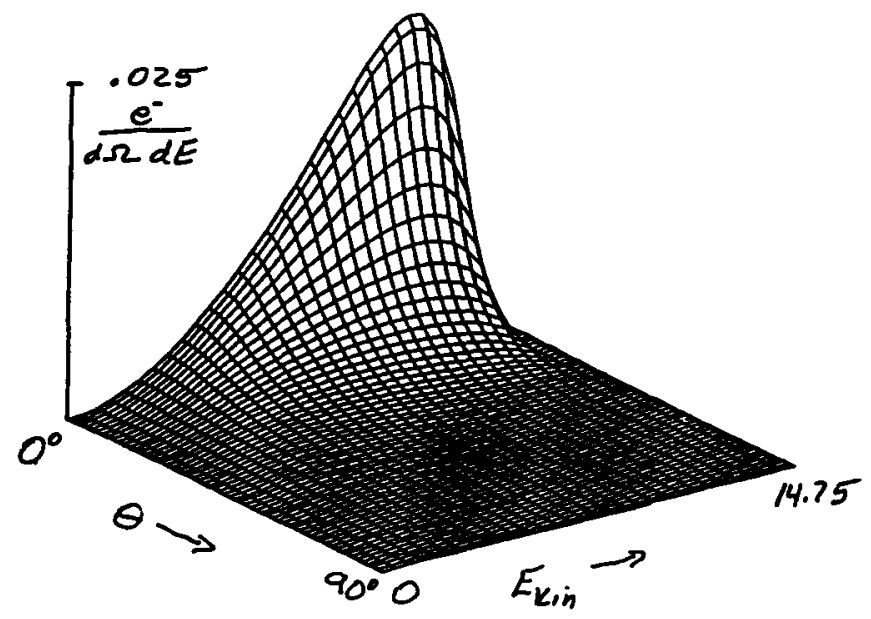

(c) $\quad N_{\text {tor }}=\int$ tot $=.095 \frac{e}{\gamma}$

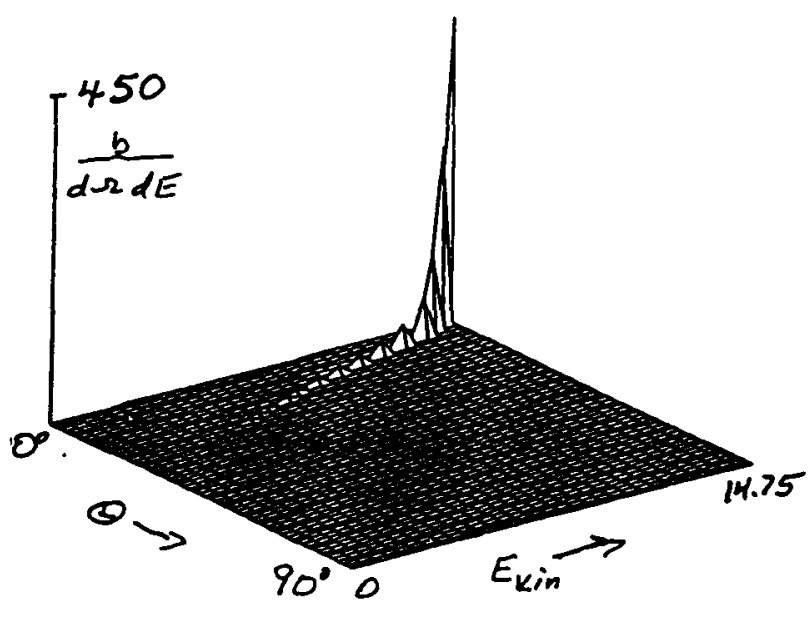

(b)

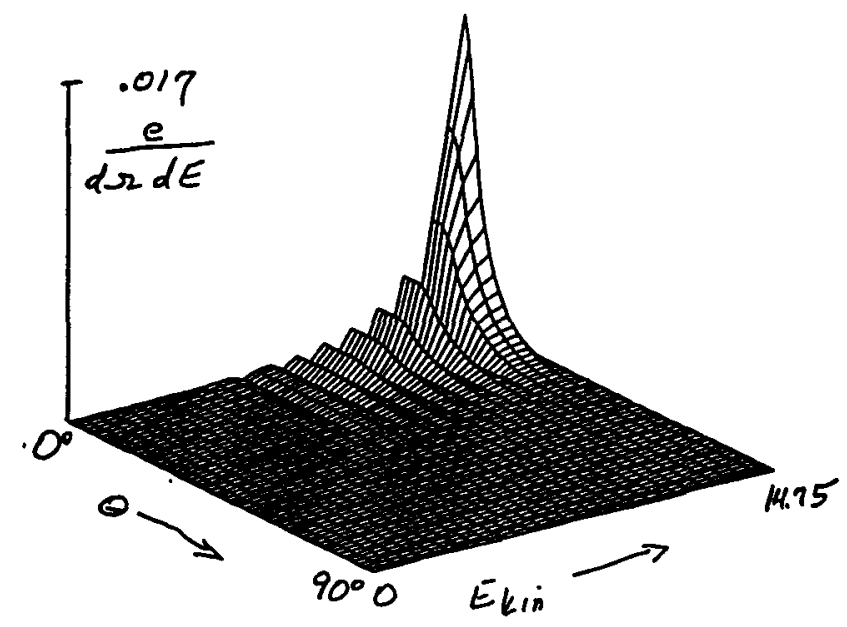

(d)

Figure 4. MOLE calculations for $E_{\gamma}=15 \mathrm{MeV}$.

Figures 4(c) and 4(d) show the Molière-scattered converter distributions. The angle bins are still $1.5^{\circ}$, but the energy bins have been increased to about 0.5 $\mathrm{MeV}$. Here, $\mathrm{N}_{\text {tot }} \approx 0.1 \mathrm{e}^{ \pm}$per $\gamma$ ray -about five times greater than for $\mathrm{E}_{\gamma}=4 \mathrm{MeV}$ (see Figs. 3(c) and 3(d)). Note that the scattered distributions in 4(c) and 4(d) are much narrower than the corresponding distributions in Figs. 3(c) and 3(d).

Figures 4(e) and (f) show results for the same "ring" collimator and Be scatterer as before. Here, $\mathrm{N}_{\text {tot }}=\mathbf{8 . 0} \cdot 10^{-3}$, gives a higher transmission efficiency through the first collimator of $8.4 \%$ (compared to $3.1 \%$ in Figs. $3(e)$ and(f) ). 

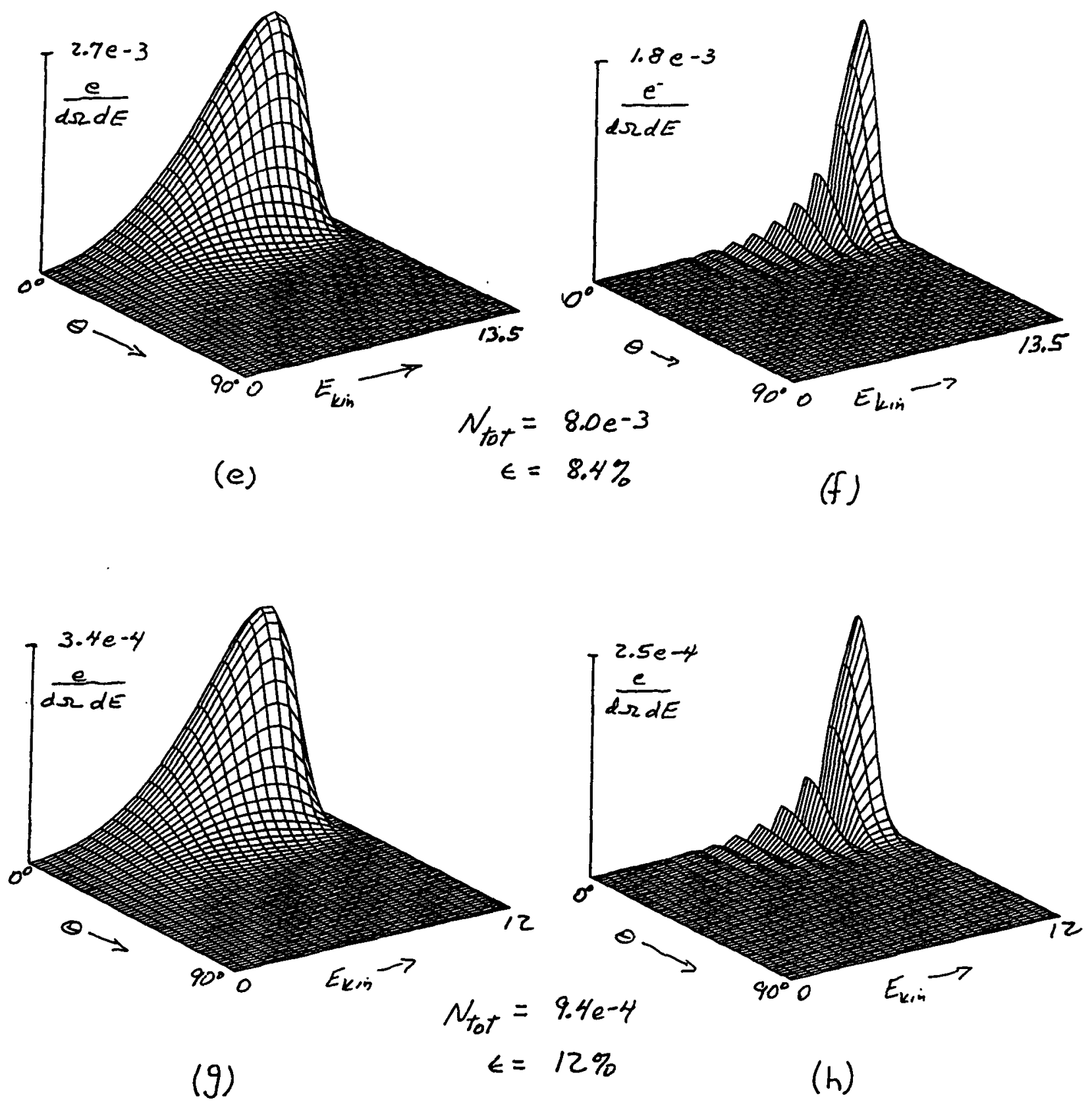

Figure 4. MOLE calculations for $E_{\gamma}=15 \mathrm{MeV}$ (cont'd).

Figure $4(\mathrm{~g})$ and $(\mathrm{h})$ show the distributions that emerge after re-collimation (on-axis) and scattering through another $0.5-\mathrm{cm} \mathrm{Be}$ scatterer, using the same physical arrangement and angular increments as before. Here, the spectra have been "ranged down" to a maximum energy of about $12 \mathrm{MeV}$. $\mathrm{N}_{\text {tot }}$ now is $9.4 \bullet 10^{-4}$, giving a transmission through this collimator of $12 \%$ (compared with $2.8 \%$ above). 


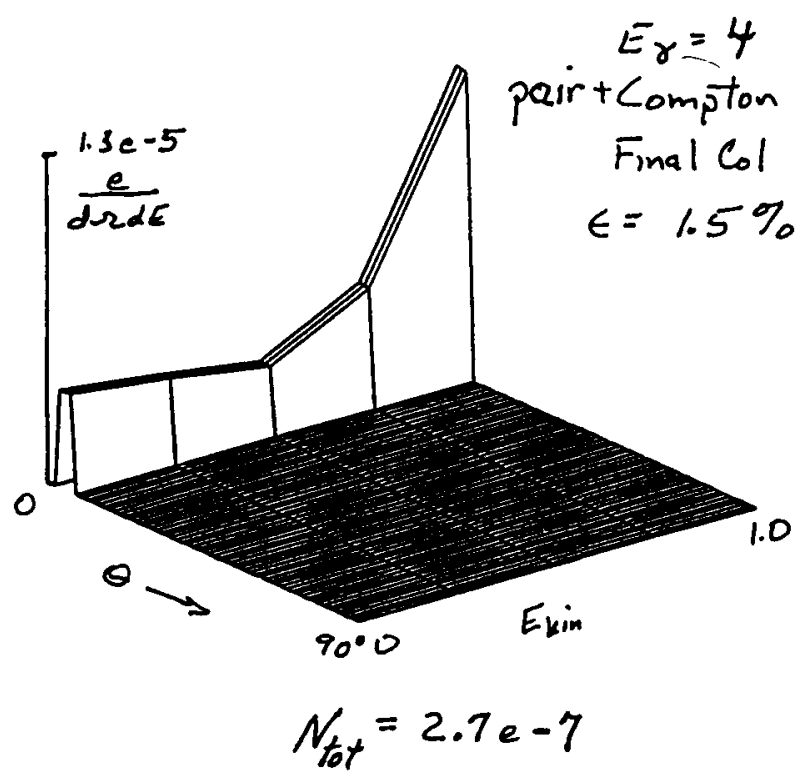

(a)

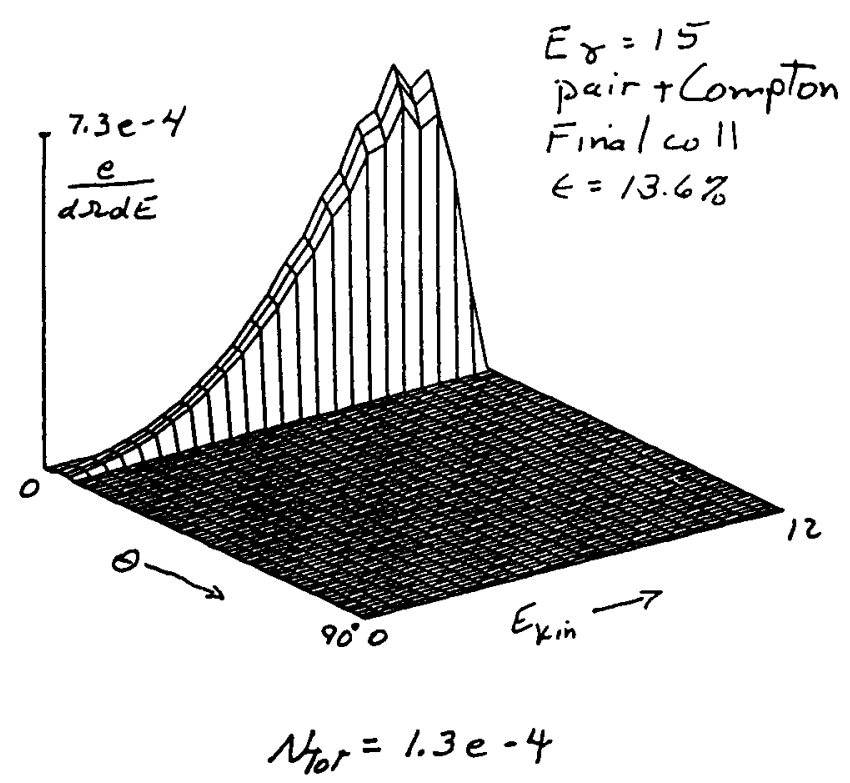

(b)

Figure 5. Final $\mathrm{e}^{ \pm}$Spectra from MOLE calculations.

Figure 5 compares the total $\mathrm{e}^{ \pm}$spectra (remember, $\mathrm{N}_{\text {tot }} \approx \mathrm{N}_{\mathrm{C}}+2 * \mathrm{~N}_{\mathrm{pp}}$ ) that emerge from the final collimator for $\mathrm{E}_{\gamma}=4 \mathrm{MeV}$ and $15 \mathrm{MeV}$. For $4 \mathrm{MeV}, 5$ (a) gives $\mathrm{N}_{\text {tot }}=2.5 \cdot 10^{-7}$, and a transmission efficiency through the final collimator of $1.5 \%$. For $15 \mathrm{MeV}, 5$ (b) gives $\mathrm{N}_{\text {tot }}=1.3 \cdot 10^{-4}$, and final transmission of $13.6 \%$.

Differences between the two final spectra are evident. For $\mathrm{E}_{\gamma}=4 \mathrm{MeV}$, the spectrum is relatively weak and is dominated by contributions from Compton scattering. For $\mathrm{E}_{\gamma}=15 \mathrm{MeV}$, the spectrum is more intense, extends to much higher energies, and is dominated by contributions from pair production.

\section{Discussion}

These calculations show the MOLE electron response to $15-\mathrm{MeV} \gamma$ rays to be 500 times more efficient than for $4 \mathrm{MeV} \gamma$ rays. Below $4 \mathrm{MeV}$, the response drops quickly to zero-there is an energy threshold at $\mathrm{E}_{\gamma} \approx 3.5 \mathrm{MeV}$. Although the 4/15-MeV discrimination might not be quite as extreme as desired, it is still quite strong, and the energy threshold should be effective for eliminating response to fluxes of lower-energy $\gamma$ rays. The $4 / 15-\mathrm{MeV}$ discrimination probably will be better than indicated here, because the calculations were conservative in several ways: energy loss in the converter is ignored, and Molière distributions were evaluated with incident particle energies, rather than "ranging" energies down continuously. 
Figure 2 shows the energy-dependence of the MOLE response from 3.5 to $20 \mathrm{MeV}$. These results were obtained from calculations that were identical to those discussed above. The overall behavior of the MOLE system is similar to the desired behavior, except that perhaps the energy discrimination isn't quite as strong as desired. At the same time, the energy threshold feature at about 3.5 $\mathrm{MeV}$ provides excellent discrimination against lower-energy $\gamma$ rays. The dotted line in Fig. 2 indicates how energy discrimination in the MOLE might be improved with a final electron detector whose response varies linearly with energy.

One type of electron detector that might prove desirable for use with MOLE is described in a recent paper. ${ }^{2}$ This detector, with a focused-Cherenkov design, has a gain of about 75 for $12-\mathrm{MeV}$ electrons. The gain probably varies at least linearly with electron energy, and even faster for energies below $5 \mathrm{MeV}$.

The basic nature of MOLE is that its relative response to lower-energy $\gamma$ rays should be much lower than for the threshold Cherenkov design. The threshold Cherenkov design suffers from signal contributions due to optical transition radiation from below-threshold electrons. The MOLE design actually stops these lower-energy electrons, thus eliminating associated signals.

The calculations above were assembled in order to obtain initial estimates of the feasibility of MOLE. Other more appropriate (but more time consuming) approaches can be used to obtain better estimates of response characteristics. Currently, initial efforts have begun to determine MOLE response characteristics with "EGS" calculations. These calculations should produce very accurate results and perhaps make possible the design of a specific experiment.

\section{Conclusions}

Preliminary calculations have been used to characterize a system designed to detect high-energy $\gamma$ rays. The system, MOLE, uses Molière scattering and other energy-dependent phenomena to discriminate against lower-energy $\gamma$ rays. The results indicate that MOLE converts high-energy $\gamma$ rays efficiently while discriminating strongly against lower-energy $\gamma$ rays, especially when an energydependent electron detector is used. 


\section{References}

1 The computer code was written by Britton Chang. The code was written with the "MATLABTM" computer code for a MacIntosh II computer. Calculations typically require several hours running time on a Mac $\mathrm{fx}$ system.

2 This detector is described in a recent paper: "A high-efficiency focusing Cherenkov radiation detector," Katina-Pilar Lewis, Michael J. Moran, and James Hall, Rev. Sci Instrum. 63, March 1992 (in press). 